\title{
e-CALLISTO Network System and the Observation of Structure of Solar Radio Burst Type III
}

\author{
M. O. Ali ${ }^{1}$, Z. S. Hamidi ${ }^{1}$, N. N. M. Shariff ${ }^{2}$, M. S. Faid ${ }^{3}$, \\ S. N. U. Sabri ${ }^{4}$, Nurulhazwani Husien ${ }^{4}$, N. H. Zainol ${ }^{4}$ \\ and C. Monstein ${ }^{5}$ \\ ${ }^{1}$ Faculty of Applied Sciences, Universiti Teknologi MARA, \\ Selangor, Malaysia \\ ${ }^{2}$ Academy of Contemporary Islamic Studies, Universiti Teknologi MARA, \\ Selangor, Malaysia \\ ${ }^{3}$ Academy of Contemporary Islamic Studies, MARA Technology University, \\ Selangor, Malaysia \\ ${ }^{4}$ Faculty of Applied Sciences, MARA Technology University, \\ Selangor, Malaysia \\ ${ }^{5}$ Insitute of Astronomy, Wolfgang-Pauli-Str, 278093 Zürich, \\ Switzerland \\ E-mail: marhanaomarali@gmail.com; \{zetysh;nnmsza\}@salam.uitm.edu.my; \\ syazwaned@siswa.um.edu.my; \{sitinurumairah; hazwani_husien21; \\ hidayahnur153\}@yahoo.com; monstein@astro.phys.ethz.ch
}

Received 20 November 2016; Accepted 20 January 2017;

Publication 10 February 2017

\section{Abstract}

This paper highlighted on the unique occurrence of the Solar Radio Burst Type III (SRBT III) during the high activities of the Sun. e-CALLISTO network is the system that responsible for the observation of the Sun 24 hours per day, which is a program under IHY/UNBSSI and ISWI instrument deployment program. The data was taken from one of the part of e-CALLISTO network which is Bleien, Switzerland. The event that had been selected was on 27th August 2015 since there was two subtypes of SRBT III can be obviously observed during the day within 12:00 UT till 12:05 UT. The current condition

Journal of Industrial Engineering and Management Science, Vol. 1, 1-14.

doi: 10.13052/jiems2446-1822.2017.001

(c) 2017 River Publishers. All rights reserved. 
of solar wind speed is $348 \mathrm{~km} / \mathrm{s}$ with density 8.4 protons $/ \mathrm{cm}^{3}$. Besides the magnetic flux also quite high which is $13.4 \mathrm{nT}$. Regarding the detection of SRBT III, the x-ray flux data from Solar Monitor shows there is strong class mflare also occur. The strong flare is also believed to have high temperature due to the high magnetic field. A geo-effective explosion was occur even though the sunspot no longer directly facing on the earth. The active region AR2403 was predicted can potentially cause radio blackout and radiation storm as long as the sunspot remains visible.

Keywords: CALLISTO network system, Solar Radio Burst, Solar Flare.

\section{Introduction}

The CALLISTO spectrometer is a programmable heterodyne receiver built in the framework of IHY2007 and ISWI by former Radio and Plasma Physics Group (PI Christian Monstein) at ETH Zurich, Switzerland [1]. CALLISTO is a network that is able to continuously observe the solar radio spectrum which is a program under IHY/UNBSSI and ISWI instrument deployment program [2]. All Callisto spectrometer all over the world form the e-Callisto network. e-CALLISTO is an acronym and stands for extended Compact Astronomical Low Cost Frequency Instrument for Spectroscopy and Transportable Observatory that is a worldwide network of frequency-agile solar spectrometer [3]. This network has a receiver instrument named CALLISTO which is inspired from one of the name Jupiter's larger moons.

This device was designed by Christian Monstein from institute for Astrophysics, ETH Zurich, Switzerland. The goal of e-Callisto is to provide

worldwide network that can monitored solar radio burst for 24 hours per day [4]. At present, more than 66 instruments have been installed at more than 35 locations, with users from more than 92 countries in the e-CALLISTO network. In this project, instrument deployment including education and training of observers was financially supported by SNF, SSAA, and NASA, institute for Astronomy and North South Center of ETH Zurich and few private sponsors. Figure 1 shows the distribution of e-CALLISTO instruments around the world [5].

CALLISTO is completed by two systems which are indoor and outdoor system. For outdoor, it has CALLISTO antenna and low-noise preamplifier. Meanwhile, indoor consist of indoor amplifier, CALLISTO Receiver, and PC. Figure 2 shows the schematic diagram of connection of CALLISTO system. The total frequency range of CALLISTO is from $45 \mathrm{MHz}$ to $870 \mathrm{MHz}$ [6]. 
e-CALLISTO Network System and the Observation of Structure 3

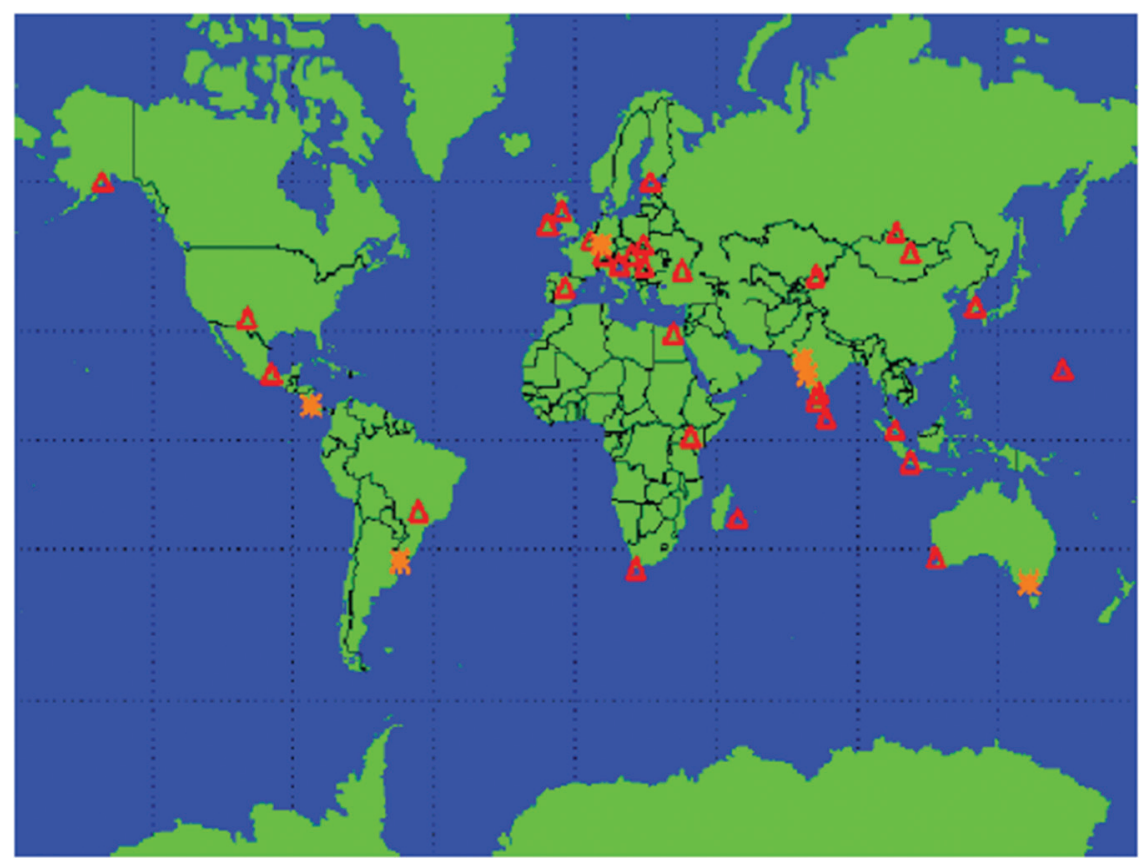

Figure 1 World CALLISTO Spectrometer network.

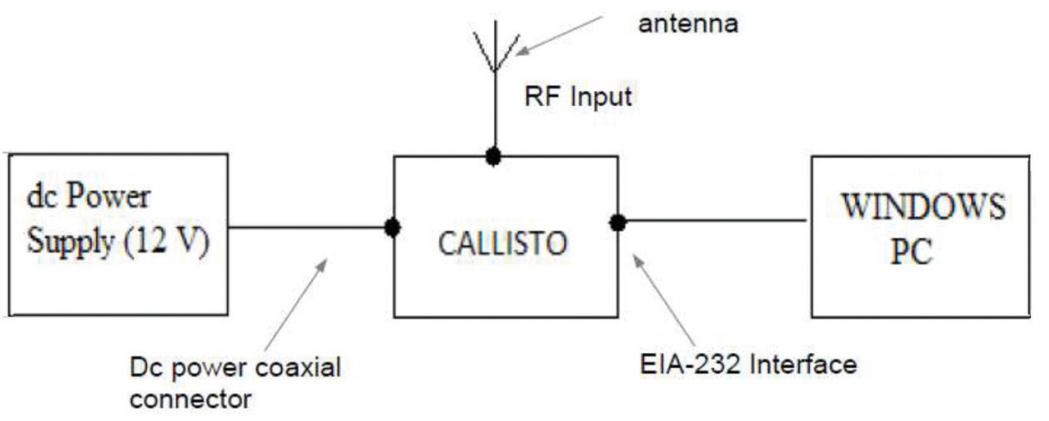

Signal from antenna

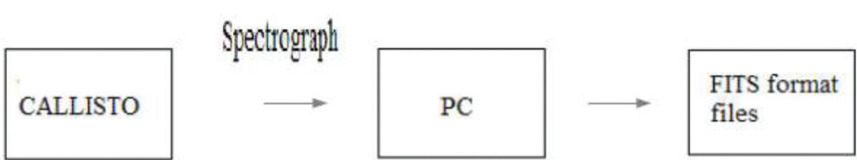

Figure 2 The schematic diagram of connection of CALLISTO system. Credit to: ROOPA Nandita PIRTHEE Sagar Girish Kumar Beeharry. 
The CALLISTO system has proven to be valuable new tool for monitoring solar activity. Besides, it has become an instrument for identifying the nature of solar radio emission from solar eruptions [7]. One of the uniqueness of the CALLISTO spectrometer network is, because of the collaboration of many countries, the Sun can be monitored 24 hours per day and all the data available online. In Figure 1, there are red triangles and orange stars. Red triangles are stations which provide data while station represented by orange stars do not provide data for several reasons.

Nowadays, the interaction between the Sun and the Earth is clearly of particular interest. A wealth of solar phenomena has been found through the Sun atmosphere such solar flare and Coronal Mass Ejections (CMEs) [8]. It is also believed that there is possibility that erratic solar effects on the Earth's climate could be very important [9]. The sun interacts with the earth through its particle emission as well as its electromagnetic radiation. During the high solar activity, the solar wind increase caused the magnetosphere of the earth disturbed thus the magnetic storm and aurora will be formed [10]. Solar wind is the situation where the charged particle, primarily electrons and protons flow out from the surface of the Sun. Space radio instrument have been designed to provide at least some information on the location of the radio source [11].

The Sun produces five different types of solar radio burst (I-IV). Type III burst one of the best indicators of release electron beams near the Sun along open magnetic field lines [12]. A solar type III radio burst is a transient burst of radio emission that starts at higher frequencies and drifts to lower frequencies as a function of time [13]. Noted that some of these type III radio bursts at kilometric wavelengths, associated with major flares, were unusually intense and had very complex and long-lasting intensity - time profiles near $1 \mathrm{MHz}$ [14]. It should also be theoretically possible to observe hard X-rays associated with the type III producing electron beams in the upper corona [15].

As mention earlier, solar flare is one of the phenomena of the Sun. Observation of solar flare in all region of electromagnetic spectrum from visible to gamma ray provides a wealth of information. Solar flare produce copious amount of coherent radio wave, which have been classified for more than 40 years into different classes [16]. Type IIIs are among the most studied solar coherent burst and generally occur at the beginning of the impulsive phase of a solar flare [17]. The x-ray and ultraviolet radiation provide evidence that locally very high temperature during flare outburst [18].

Solar flare that has the most significant effect on earth is the largest solar flare named X-class flares [19]. Some of the effects are long lasting radiation storms in the upper atmosphere and trigger blackouts. There are also other type of flare which is Medium size M-class flares which can cause brief radio 
blackouts in the polar region and the occasional minor radiation storms [20]. Meanwhile, C-class flares have few noticeable consequences. Since X-class flare is the largest solar flare, absorbing it will affects the atmosphere. It will results in increase in heat and an expansion of the Earth ionosphere [21].

It is believed that solar activities such as and solar flare have a very close connection and significant impact of the climate changes and Earth's environment [5]. The value of solar radio bursts at low frequencies lies in the fact that they originate in the same layers of the solar atmosphere in which geoeffective disturbances probably originate: the layers where energy is released in solar flares, where energetic particles are accelerated, and where coronal mass ejections (CMEs) are launched [22]. The dynamical behaviour of the Sun exhibits a variety of physical phenomena, some of which are still not at all or only barely understood due to the complexity of the structure of the Sun [23].

\section{Methodology}

In this study, the data were taken from Bleien's Callisto Spectrometer since the data taken was really clear with less noise as compared to the other spectrometer and the maintenance of the spectrometer is really good. Besides, some information were also taken from space agency such National Aeronautics and Space Administration (NASA), Solar Monitor, Space Weather Prediction Centre and SOHO Observatory that make their data available online so that the observation can be made more detail.

Figure 3 shows the Long Wavelength Antenna (LWA) in Bleien and it Callisto coverage. This antenna covers the frequency range 45 till $870 \mathrm{MHz}$ that provide a very good data of Solar Radio Burst. The maximum coverage that Bleien has is within May to Jun which the data of Solar Radio Burst available start from 03:00 UT to 19:00 UT. Basically, the signal from the feed will be fed into the receivers and it will be converted to a first intermediate frequency of $37.7 \mathrm{MHz}$ by two local oscillators. The event that had been selected was on 27th August 2015 since there was two subtypes of SRBT III can be obviously observed during the day within 12:00 UT till 12:05 UT.

\section{Result and Analysis}

Figure 4 shows complex and single type III Solar Radio Burst were observed on 27th August 2015 which both of them occur within 12:00 UT to 12:05 UT and the sunspot active region (AR) is AR2403. It is unique because two types 


\section{M. O. Ali et al.}
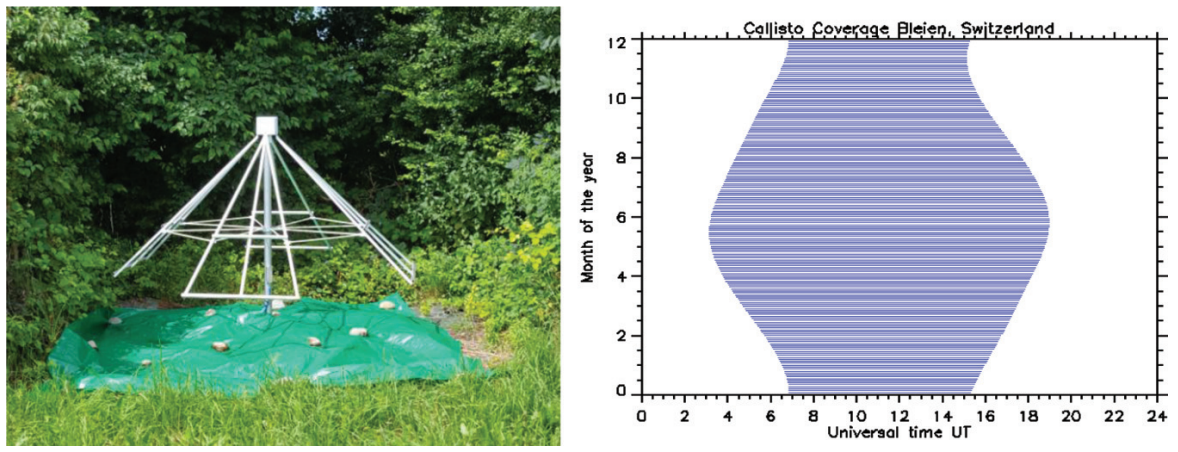

Figure 3 Long wavelength antenna and Callisto coverage of Blein Switzerland.

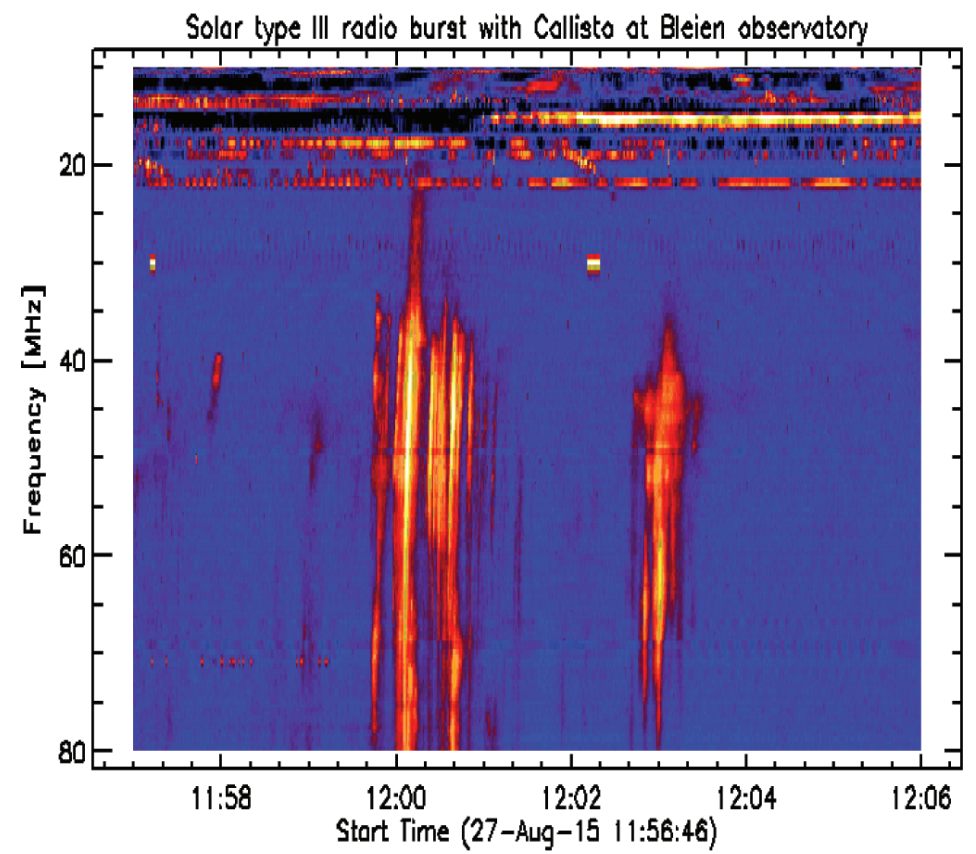

Figure 4 Solar radio burst type III on 27th August 2015

of subtypes SRBT III occur continuously after one another. For the complex SRBT III it was drifted from $20 \mathrm{MHz}$ to $80 \mathrm{MHz}$. meanwhile for the single SRBT III it was drifted from $35 \mathrm{MHz}$ to $80 \mathrm{MHz}$. The duration for the first SRBT III is approximately one minute and the second SRB only about 30 seconds. 
Table 1 Table of condition of space weather on 27th August 2015

\begin{tabular}{ll}
\hline Solar Wind's Speed & $348 \mathrm{~km} / \mathrm{s}$ \\
Proton density & $8.4 \mathrm{protons} / \mathrm{cm}^{3}$ \\
Interplanetary mag. field & $13.4 \mathrm{nT}$
\end{tabular}

During the day, the space weather were show in Table 1.

It was observed that the interplanetary magnetic field really high which is $13.4 \mathbf{n T}$. Since magnetic reconnection was the primary energy release mechanism in solar phenomena thus the higher the magnetic field the higher the temperature of the flare. This relationship can be confirmed by the relation (1). Whereby $\boldsymbol{B}$ is magnetic field, $n_{0}$ is proton density, and $\boldsymbol{L}$ height of flare.

$$
T_{\text {flare }}=3 \times 10^{7}\left(\frac{\boldsymbol{B}}{50 \mathrm{G}}\right)^{\frac{6}{7}}\left(\frac{n_{0}}{10^{9} \mathrm{~cm}^{-3}}\right)^{\frac{-1}{7}} \times\left(\frac{\boldsymbol{L}}{10^{9} \mathrm{~cm}}\right)^{\frac{2}{7}} K
$$

Figure 5 shows the x-ray flux data for 25th August till 28th August 2015. It can be observed that the highest class of flare was on 27th August 2015.

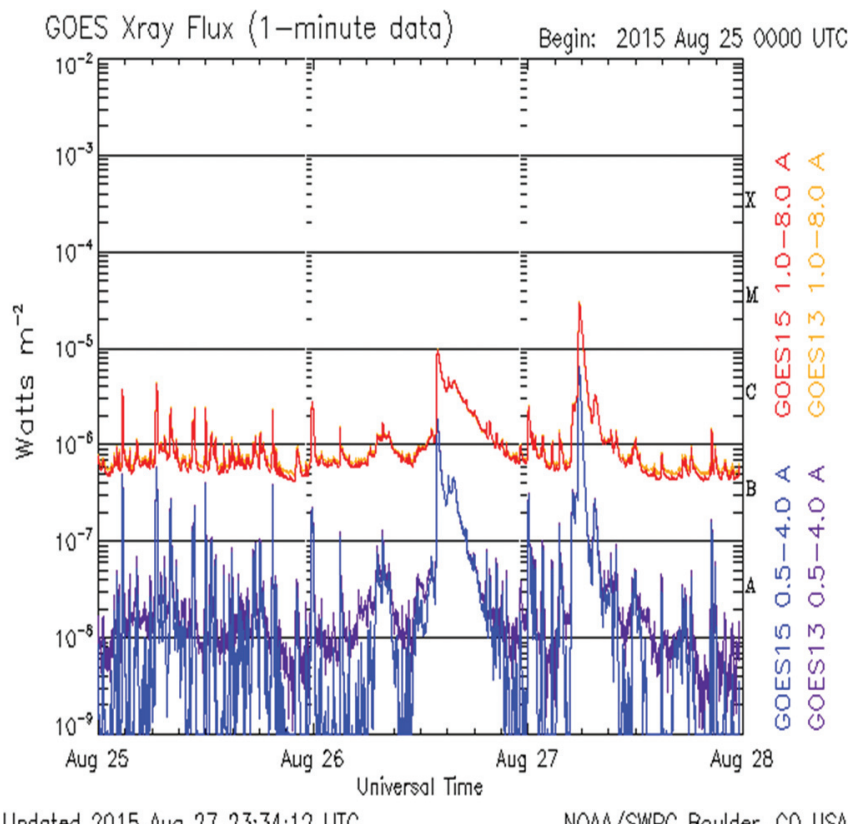

Updated 2015 Aug 27 23:34:12 UTC

NOAA/SWPC Boulder, CO USA

Figure 5 X-ray flux data for 25th till 28th August 2015. 
The day before, 26th August the x-ray flux data shows the flare on that day C9 class flare. It indicates that the flare can be even bigger.

Space Weather predicted that there might be a huge flare on 27th August 2015 due to the high interplanetary magnetic field and high flare temperature. The x-ray flux data from solar monitor shows that M2 class of flare occurred during the day. $\mathrm{M}$ class flare was considered as a strong flare after $\mathrm{X}$ class flare which also can caused impact to the earth. Sunspot AR403 was observed approaching the limb of sun. A geo-effective explosion was happen even though the sunspot no longer directly facing on the earth. AR2403 was predicted can potentially cause radio blackout and radiation storm as long as the sunspot remains visible.

\section{Conclusion}

Solar Radio Burst Type III is one of the best indicator for the solar flare event that occurred during the high solar activities. The powerful of the explosion or the flare are related to the magnetic field of the active region of the Sun through the process of magnetic reconnection. The stronger the magnetic field of the active region, the higher the temperature of the flare thus resulting in high class of flare. A continuous observation on the Sun is critical since it affects the condition of the earth and sometimes will affect the high technology activities. If the impact of the flare is only a minor impact it will caused the beautiful aurora.

\section{Acknowledgment}

We are grateful to CALLISTO network, NASA, STEREO, LASCO, SDO/ AIA, NOAA and SWPC make their data available online. This work was partially supported by the 600-RMI/FRGS 5/3 (135/2014) and 600-RMI/RAGS 5/3 (121/2014) UiTM grants and Kementerian Pengajian Tinggi Malaysia. Special thanks to the National Space Agency and the National Space Centre for giving us a site to set up this project and support this project. Solar burst monitoring is a project of cooperation between the institute for Astronomy, ETH Zurich, and FHNW Windisch, Switzerland, Universiti Teknologi MARA and University of Malaya. This paper also used NOAA Space Weather Prediction Centre (SWPC) for the sunspot, radio flux and solar flare data for comparison purpose. The research has made use of the National Space Centre Facility and a part of an initiative of the International Space Weather Initiative (ISWI) program. 


\section{References}

[1] Benz, A. O., Monstein, C., and Meyers, H. (2004). CALLISTO, A New Concept for Solar Radio Spectrometer. The Netherland: Kluwer Academic Publishers.

[2] Benz, A. O., Guedel, M., Isliker, H., Miszkowicz, S., and Stehling, W. (1991). A broadband spectrometer for decimetric and microwave radio bursts first results. Sol. Phys. 133, 385-393.

[3] Hamidi, Z., Shariff, N., Abidin, Z., Ibrahim, Z., and Monstein. C. (2013). E-callisto collaboration: some progress solar burst studies associated with solar flare research status in Malaysia, Malays. J. Sci. Technol. Stud. 9, 15-22.

[4] Hamidi, Z., Shariff, N., Abidin, Z., Ibrahim, Z., and Monstein, C. (2012). Coverage of solar radio spectrum in Malaysia and spectral overview of radio frequency interference (RFI) by using CALLISTO spectrometer from $1 \mathrm{MHz}$ to $900 \mathrm{MHz}$. Middle-East J. Sci. Res. 12, 893-898.

[5] Hamid, Z. S., Ungku Ibrahim, U. F. S., Abidin, R., and Shariff, N. N. M. (2013). Theoretical Review of Solar Radio Burst III (SRBT III) Associated With of Solar Flare Phenomena. Int. J. Fundam. Phys. Sci. 3, 20-23.

[6] Benz, A. O., Monstein, C., and Meyer, H. (2005). Callisto - a new concept for solar radio spectrometers. Sol. Phys. 226, 143-151.

[7] Zawari, A., Islam, M. T., Anwar, R., Hasbi, A. M., Asillam, M. F., and Monstein, C. (2014). Callisto radio spectrometer construction at universiti kebangsaan malaysia [antennas and propagation around the world]. IEEE Antennas and Propag. Mag. 56, 278-288.

[8] Gopalswamy, N., Yashiro, S., Liu, Y., Michalek, G., Vourlidas, A., Kaiser, M. L., and Howard, R. A. Coronal mass ejections and other extreme characteristics of the 2003 October-November solar eruptions. J. Geophys. Res. Space Sci. 110, A09S15-JA010958.

[9] Kruger, A. (1979). Introduction to Solar Radio Astronomy and Radio Physics. Dordrecht: D. Reidel, Publ. Co.

[10] Mann, G. (1995). Coronal Magnetic Energy Releases. Berlin: Springer.

[11] McLean, D. J. and Labrum, N. R. (eds). (1985). Solar Radiophysics. Cambridge: Cambridge University Press.

[12] Lobzin, V. V., Cairns, I. H., Robinson, P. A., Steward, G., and Patterson, G. (2009). Automatic recognition of type III solar radio bursts; automated radio burst identification system method and first observations. Space Weather. 7, 1-12. 
[13] Reale, F. (2010). Coronal loops: observations and modeling of confined plasma. Living Rev. Sol. Phys. 7, 5.

[14] Kundu, M. R. (2002). Radio observations of high energy solar flares. Highl. Astron. 12, 379-383.

[15] Gopalswamy, N. (2000). Radio-rich solar eruptive events. Geophys. Res. Lett. 27, 1427-1430.

[16] Benz, A. O. (2008). Flare observations. Living Rev. Sol. Phys. 5, 1.

[17] Dulk, G. A. (2000). "Type III solar radio bursts at long wavelengths," in Geophys. Monogr, eds. R. Stone, E. Weiler, and M. Goldstein. Washington, DC: American Geophysical Union.

[18] Belov, A., Garcia, H., Kurt, V., and Mavromichalaki, E. (2005). Proton events and X-ray flares in the last three solar cycles. Cosmic Res. 43, $165-178$.

[19] Chubb, T. A., Kreplin, R. W., and Friedman, H. (1966). Observations of hard X-ray emission from solar flares. J. Geophys. Res. 71, 3611-3622.

[20] Cairns, I. H. and Knock, S. A. (2002). Predictions for dynamic spectra and source regions of type II radio bursts In the inhomogenous corona and solar wind. Sol. Phys. 210, 419-430.

[21] Kahler, S. W. (1992). The role of the big flare syndrome in correlations of solar energetic proton fluxes and associated microwave burst parameters. J. Geophys. Res. 87, 3439-3448.

[22] Krucker, S. and Lin, R. P. (2000). Two classes of solar proton events derived from onset time analysis. Astrophys. J. 542, 61-64.

[23] Hamidi, Z., Ibrahim, U., Salwa, U. F., Abidin, Z., Ibrahim, Z., and Shariff, N. (2013). Theoretical Review of Solar Radio Burst III (SRBT III) Associated with of Solar Flare Phenomena. Int. J. Fundam. Phys Sci. 3, 20. 


\section{Biographies}

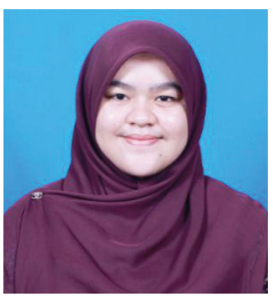

M. O. Ali is currently a master student at Universiti Teknologi MARA, Shah Alam Malaysia. Interest in study of solar radio burst type III and solar flare.

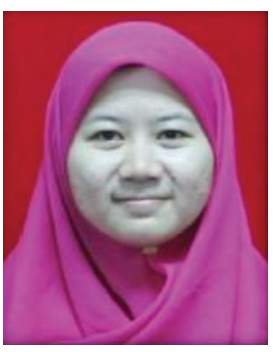

Z. S. Hamidi is currently a Senior Lecturer at Universiti Teknologi MARA, Shah Alam Malaysia. Involve in solar physics research and the project is under International Space Weather Initiative (ISWI) project under NASA project and published more than 150 publications. Also involves in others area of astronomy.

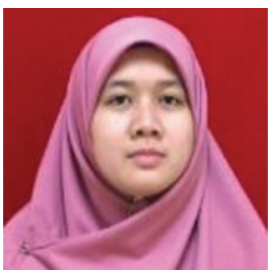

N. N. M. Shariff is a senior lecturer at Academy of Contemporary Islamic Studies, Shah Alam. Currently she holds several positions as a Research Management Unit (RMU) coordinator and Malaysian Research Assessment (MyRA) Liaison Officer. She is one of editorial board for Journal of Contemporary Islamic Studies (JCIS), Senior Fellow of Centre for Human Rights and Advocacy (CENTHRA) and member of Malaysian Islamic Astronomy Society. 


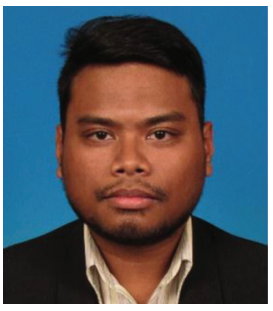

M. S. Faid is currently a master student at University Teknologi MARA Malaysia.

Researching in algorithm for new moon visibility criteria. Interest in study of light pollution and twilight sky brightness.

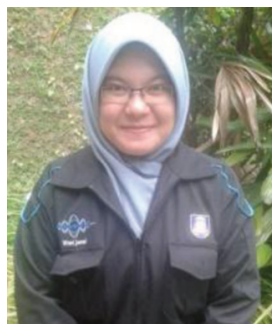

S. N. U. Sabri is currently a master student at Universiti Teknologi MARA, Shah Alam Malaysia. Involve in investigation on evaluation beta-gamma magnetic field on active region in solar radio burst.

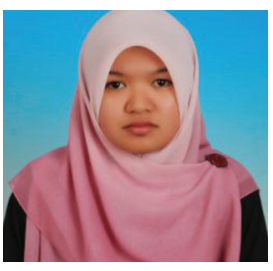

N. H. Husein is currently a master student at Universiti Teknologi MARA, Shah Alam Malaysia. Researching in solar radio burst type II and III related with CME and solar flare. 


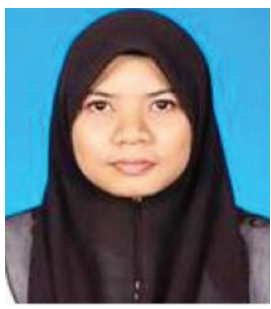

N. H. Zainol is currently a master student at Universiti Teknologi MARA, Shah Alam Malaysia. Study the Coronal Mass Ejection Based of Moreton Waves on the Characteristic of Solar Radio Burst Type II.

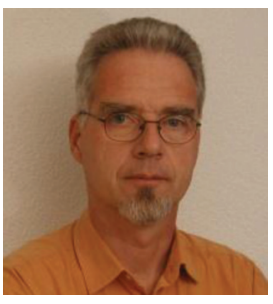

C. Monstein is a native of Switzerland and lives in Freienbach. He obtained Electronics Engineer, B.S. degree at Konstanz University, Germany. He has 19 years' experience designing automatic test systems in the telecommunications industry and is proficient in several programming languages. He presently works at ETH-Zürich on the design of digital radio spectrometers (frequency agile and FFT) and is responsible for the hardware and software associated with the e-CALLISTO Project. He also has participated for 10 years in the European Space Agency space telescope Herschel (HIFI), 6 years in the European Southern Observatory project MUSE for VLT in Chile, and 2 years in NANTEN2 (delivery of the FFT radio spectrometers for the Submillimeter Observatory at Pampa la Bola, Chile). Currently he is quite involved in commissioning the correlation receiver at the $7 \mathrm{~m}$ radio telescope in Switzerland, foreseen as a test bed for the cosmologists. He is a member of the European Committee for Radio Astronomy Frequencies (CRAF) and he is also a member of ISWI steering committee at United Nations Office for Outer space Affairs (UNOOSA) in Vienna. He is also a member of IEEE and he plays also the role of a coordinator of SetiLeague in Switzerland and is licensed as amateur radio operator, HB9SCT. 
